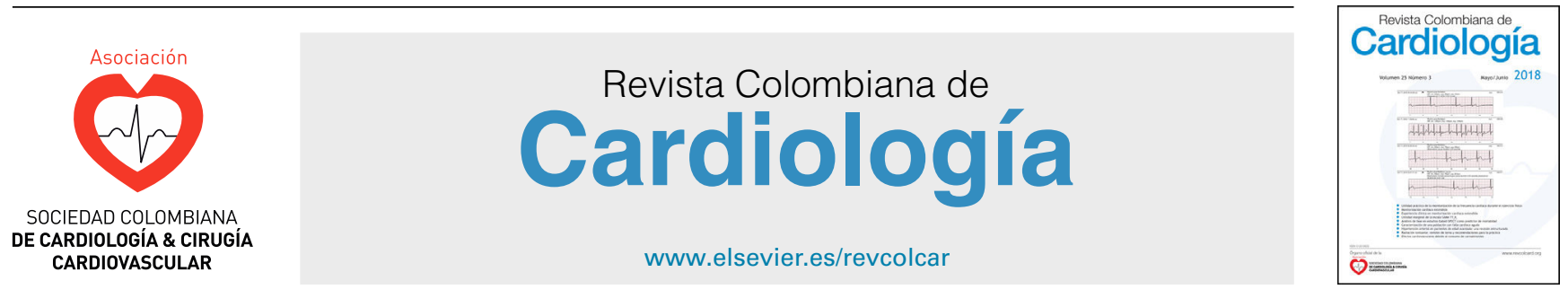

CARDIOLOGÍA DEL ADULTO - PRESENTACIÓN DE CASOS

\title{
Ablación de reentrada nodal en vena cava superior izquierda persistente y ausencia de vena cava superior derecha
}

\author{
Miguel J. Tejeda-Camargo ${ }^{\mathrm{a}, \mathrm{b}, *}$, Alonso Arroyo ${ }^{\mathrm{a}, \mathrm{b}}$, Carlos Tapias $^{\mathrm{b}}$ y Diego Rodríguez ${ }^{\mathrm{a}, \mathrm{b}}$ \\ a Centro internacional de arritmias "Andrea Natale", Fundación Cardioinfantil-Instituto de Cardiología, Bogotá, Colombia \\ b Universidad de la Sabana, Bogotá, Colombia
}

Recibido el 20 de mayo de 2019; aceptado el 15 de agosto de 2019

Disponible en Internet el 25 de noviembre de 2019

\section{PALABRAS CLAVE \\ Taquicardia \\ paroxística; \\ Taquicardia por \\ reentrada en el nodo \\ atrioventricular; \\ Vena cava superior; \\ Anomalías congénitas}

\section{KEYWORDS}

Paroxysmal

tachycardia;

Atrioventricular

nodal re-entry;

Superior cava vein;

Congenital

abnormalities

\begin{abstract}
Resumen La taquicardia por reentrada nodal es la arritmia más frecuente entre las taquicardias supraventriculares paroxísticas; la variedad lenta-rápida es la más común. En muchos casos la ablación de la vía lenta es el tratamiento definitivo y puede llegar a tener alta tasa de éxito y baja frecuencia de complicaciones. La presencia de una vena cava superior izquierda y la ausencia de vena cava superior derecha con drenaje venoso sistémico superior al seno coronario es una malformación congénita poco frecuente, la cual genera alteraciones anatómicas que dificultan el abordaje tradicional para la ablación de esta arritmia. Se presenta el caso de una paciente con esta condición en la que fue exitosa la ablación mediante ecocardiografía intracardiaca y reconstrucción tridimensional.

( 2019 Publicado por Elsevier España, S.L.U. en nombre de Sociedad Colombiana de Cardiología y Cirugía Cardiovascular. Este es un artículo Open Access bajo la licencia CC BY-NC-ND (http: / / creativecommons.org/licenses/by-nc-nd/4.0/).
\end{abstract}

Nodal re-entry in patient with persistent left superior vena cava and absent right superior vena cava

\begin{abstract}
Nodal re-entrant tachycardia is the most common arrhythmia among the paroxysmal atrioventricular tachycardias, with the slow-fast variant being the most common. In many cases, the ablation of the slow pathway is the definitive treatment in many cases, often with a high rate of success and low frequency of complications. The presence of a left superior vena cava and the absence of a right superior vena cava with systematic venous drainage above the coronary sinus is a rare congenital malformation that leads to anatomical anomalies.
\end{abstract}

\footnotetext{
* Autor para correspondencia.

Correo electrónico: migueljosetejeda@hotmail.com (M.J. Tejeda-Camargo).
} 
These make it difficult to use the traditional approach for the ablation of this arrhythmia. The case is presented of a patient with this condition in which the ablation was successful using intracardiac echocardiography and three-dimensional reconstruction.

(c) 2019 Published by Elsevier España, S.L.U. on behalf of Sociedad Colombiana de Cardiología y Cirugía Cardiovascular. This is an open access article under the CC BY-NC-ND license (http:// creativecommons.org/licenses/by-nc-nd/4.0/).

\section{Introducción}

Las arritmias son causa frecuente de consulta; algunas de estas se presentan por episodios paroxísticos de palpitaciones y cuando se hacen sostenidas muchas veces obligan a consultar al servicio de urgencias. Entre las taquicardias supraventriculares, la taquicardia por reentrada nodal es la más habitual, en cuyo caso el tratamiento médico y la ablación con catéter son las alternativas racionales de tratamiento. En pacientes sintomáticos, refractarios al manejo médico y aquellos que no deseen consumo de medicación antiarrítmica a largo plazo la ablación con catéter constituye la alternativa más eficaz para el tratamiento de esta condición dada la alta tasa de éxito y la baja frecuencia de complicaciones (el bloqueo AV es menor del $0,8 \%{ }^{1}$; es esta la complicación más importante). En la mayoría de los casos la ablación es guiada por fluoroscopia y señales de electrogramas endocavitarios; sin embargo, este abordaje supone una anatomía usual.

Las alteraciones anatómicas pueden traducirse en ablación inefectiva o en muchos casos requerir tecnología adicional, como la ecocardiografía intracardiaca (EIC) y los sistemas de mapeo tridimensional (MTD), para asegurar el éxito del procedimiento gracias a la baja tasa de complicaciones.

A continuación se reporta el caso de una paciente con evidencia de taquicardia supraventricular paroxística y alta sospecha clínica de reentrada intranodal en la que se hizo mapeo y ablación tridimensional por presencia de vena cava superior izquierda persistente y ausencia de vena cava superior derecha.

\section{Caso}

Paciente femenina, de 45 años, sin antecedentes patológicos relevantes, quien acudió al servicio de urgencias por cuadro clínico de un año de evolución de palpitaciones recurrentes, ocasionalmente acompañadas de disnea, disconfort torácico y además sensación de latido de gran intensidad en el cuello (signo de la rana). Dada la persistencia de los síntomas se ordenó electrocardiograma que documentó taquicardia regular de complejo estrecho, sin evidencia de onda $P$ en el trazado, hallazgos sugestivos de taquicardia por reentrada nodal de la variedad común (lento-rápida) como mecanismo subyacente de la arritmia. Se ordenó hemograma, función tiroidea y electrolitos los cuales fueron normales. Un ecocardiograma transtorácico realizado en el servicio de urgencias reveló una dilatación del seno coronario y por lo demás un corazón estructuralmente sano. Fue llevada inicialmente a mapeo y ablación convencional, la cual fue fallida por dilatación del seno coronario, inestabilidad marcada del catéter de ablación y hallazgos en venografía que sugirieron probable diagnóstico de vena cava superior izquierda persistente. Teniendo en cuenta la frecuencia de los síntomas y el no deseo de continuidad de la medicación a largo plazo se ordenó nuevo mapeo y ablación de la arritmia. Se tuvieron en consideración los hallazgos descritos en el ecocardiograma transtorácico, la venografia y la inestabilidad del catéter en el mapeo convencional por lo que se solicitó nuevo procedimiento bajo reconstrucción tridimensional y EIC.

Se hizo abordaje tradicional con punción venosa bilateral femoral a través de la cual se ascendieron catéteres de registro y ablación, así como catéter de EIC. Se inyectó medio de contraste en miembro superior derecho para venografía (fig. 1) identificando flujo a través de la vena subclavia derecha, innominada, cava superior izquierda drenando al seno coronario y ausencia de la vena cava superior derecha; de esta manera se confirmó la sospecha inicial.

El estudio electrofisiológico con estimulación auricular y un extraestímulo indujo taquicardia regular de complejo estrecho con VA septal de $12 \mathrm{~ms}$, VH largo y HA corto, que sugirió taquicardia por reentrada nodal típica. La taquicardia terminó de manera espontánea con activación auricular, lo cual sugirió bloqueo del nodo AV (fig. 1). A través de guía ecocardiografíca se hizo reconstrucción tridimensional de la aurícula derecha, el seno coronario y la vena cava superior izquierda. Se identificó el His (se hizo nube del His) y se procedió con la ablación (fig. 2) de vía lenta nodal (guía anatómica y por electrogramas). El procedimiento fue exitoso y no se registró recurrencia de la taquicardia a seis meses de seguimiento.

\section{Discusión}

La taquicardia por reentrada nodal es la taquicardia supraventricular paroxística más frecuente; constituye así más del $60 \%$ de los $\operatorname{casos}^{2}$ y tiene predilección por el género femenino $^{3}$. La ablación con catéter a través de radiofrecuencia o crioablación tiene altas tasas de éxito (90 a 97\% de probabilidad de curación) y baja probabilidad de complicaciones ${ }^{4}$. Desde el punto de vista fisiopatológico, la mayoría de taquicardias por reentrada nodal ocurren en pacientes que tienen doble fisiología (vía de conducción lenta y rápida) nodal ${ }^{5}$. En la formación del circuito existen tres variedades: lento-rápida (denominada típica), 


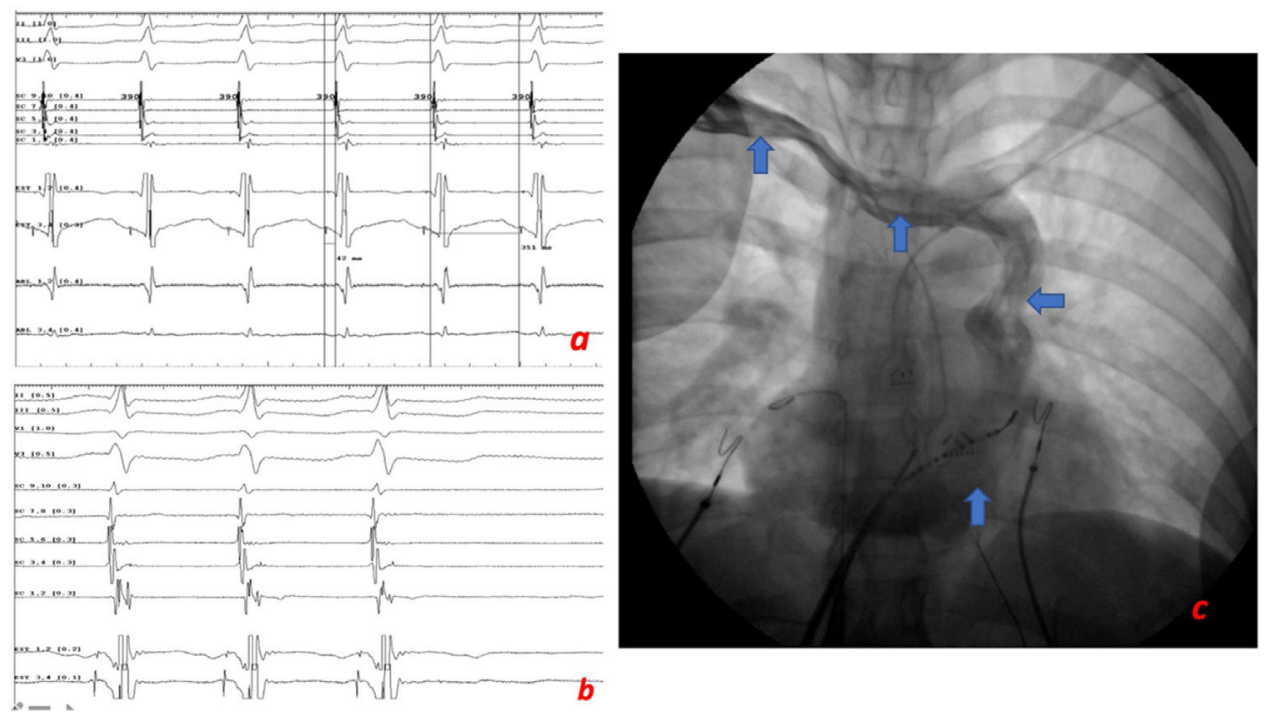

Figura 1 A. Taquicardia clínica VA 12 ms descartando AVRT, con VH largo y HA corto que sugiere AVRNT típica. B. Taquicardia espontánea que termina con la activación auricular, sugiriendo bloqueo en el nodo AV. C. Proyección de oblicua anterior izquierda; venografía desde el brazo derecho que muestra el flujo (flechas azules) de la vena subclavia derecha, la vena innominada, la vena cava superior izquierda y el seno coronario; también la venografía señala la ausencia de vena cava superior derecha.
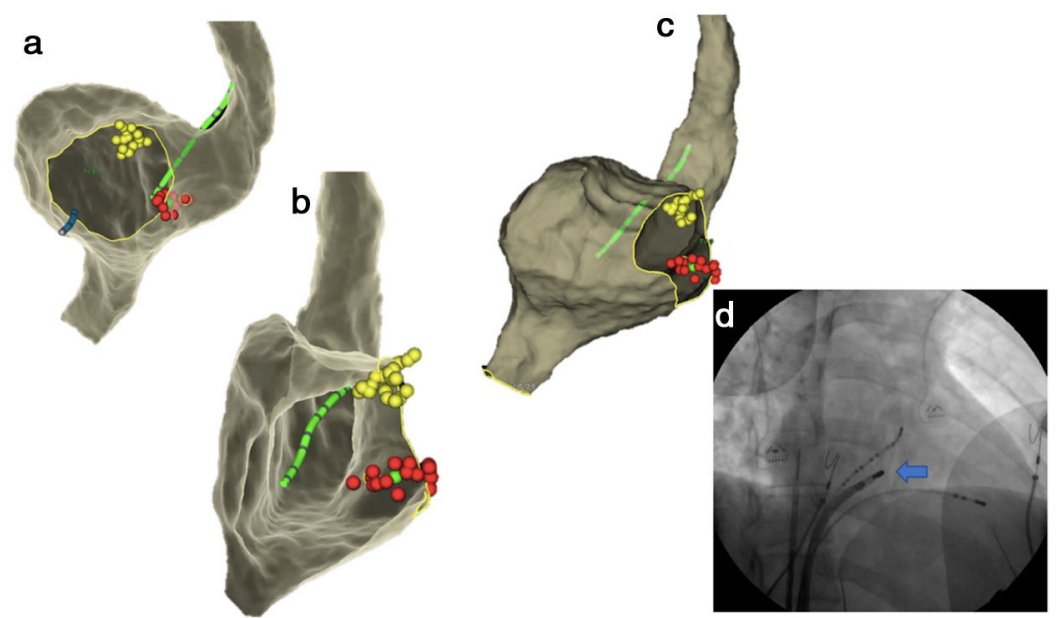

Figura 2 A. Reconstrucción 3D de la aurícula derecha en proyección oblicua anterior izquierda; los puntos amarillos indican electrogramas del His y los puntos rojos de ablación. B. Vista endoscópica tridimensional del seno coronario. C. Reconstrucción tridimensional del seno coronario en la proyección oblicua anterior derecha. D. Proyección oblicua anterior derecha que muestra la ubicación del catéter y el punto de ablación.

rápido-lenta y lento-lenta. La forma "típica" es el mecanismo más frecuente de la arritmia en un $90 \%$ aproximadamente. La eliminación de la conducción 1:1 sobre la vía lenta es el objetivo de la ablación en todas las formas de taquicardia por reentrada nodal ${ }^{6}$. En la selección del sitio de ablación existen dos aproximaciones complementarias entre si, una guía anatómica que se basa en la localización del catéter de ablación entre el ostium del seno coronario y el anillo de la válvula tricúspide, alejando el catéter de la parte superior del triangulo de Koch por aumento del riesgo de bloqueo aurícula-ventricular; en esta zona generalmente el electrograma ventricular es mayor que el auricular, ademas de lo anterior un segundo abordaje se concentra en identificar potenciales de activación de la vía lenta en esta área anatomica ${ }^{5}$.

Las alteraciones anatómicas resultantes de malformaciones congénitas pueden dar como resultado una mayor complejidad en cuanto a la práctica de los procedimientos e influenciar directamente el posicionamiento, la estabilidad y el contacto del catéter e impactar así el éxito de la ablación y el número de complicaciones potenciales resultantes del procedimiento.

La vena cava superior izquierda persistente es la malformación venosa del sistema torácico más frecuente ${ }^{7}$; afecta aproximadamente 0,3 a $0,5 \%$ de la población general ${ }^{8}$. La incidencia antes descrita se incrementa ante otras 
malformaciones cardiacas; sin embargo, la asociación entre la vena cava superior izquierda persistente y la ausencia de vena cava superior derecha (condición conocida como vena cava superior izquierda persistente aislada) es mucho menos común $(0,07-0,13)$ y hasta la mitad de estos pacientes tienen otras condiciones como defectos del tabique interventricular ${ }^{8}$. Desde el punto de vista embriológico un fracaso en la vena cardinal anterior izquierda da como resultado esta anomalía congénita ${ }^{9}$. En cuanto a la clínica es generalmente asintomática y los hallazgos electrocardiográficos pueden estar en relación con crecimiento auricular derecho. El hallazgo más frecuente a nivel ecocardiográfico es la dilatación del seno coronario en el eje paraesternal largo, el cual se puede confirmar a través del uso de solución salina agitada. La dilatación del seno coronario podría condicionar alteraciones de la arquitectura local que llevan a una propensión por arritmias reentrantes ${ }^{7,10,11}$.

Existen pocos casos publicados en la literatura acerca de estrategias de ablación en esta condición en particular, la mayoría de los cuales son antiguos y utilizaron como aproximación la fluoroscopia, así como los registros de electrogramas, sin dejar claro cuál es la metodología idónea que lleve a un balance entre el éxito y el riesgo de complicaciones teniendo en cuenta la alteración anatómica (dilatación del seno coronario) secundaria observada en esta malformación congénita.

Este caso constituye uno de los primeros reportados en cuanto a asociación entre vena cava superior izquierda aislada y taquicardia por reentrada nodal de la variedad común.

Se propone una aproximación multimodal con la utilización de EIC y sistema de MTD para una ablación más segura, con mayor probabilidad de éxito y menor riesgo de complicaciones.

\section{Conclusiones}

La vena cava superior izquierda persistente es la malformación congénita venosa torácica más frecuente; no obstante, la ausencia de vena cava superior derecha persistente aislada es una asociación poco común. Se expone el caso de una paciente con taquicardia por reentrada nodal en quien se logra una ablación efectiva haciendo énfasis en la importancia del enfoque imagenológico multimodal en este tipo de pacientes en quienes la EIC y los sistemas de MTD desempeñan un rol importante ya que favorecen el posicionamiento, el contacto y la estabilidad del catéter de ablación.

\section{Financiación}

Ninguna.

\section{Conflicto de intereses}

Ninguno.

\section{Bibliografía}

1. Chen H, Shehata M, Ma W, Xu J, Cao J, Cingolani E, et al. Atrioventricular block during slow pathway ablation: entirely preventable? Circ Arrhythm Electrophysiol. 2015;8: 739-44.

2. Ganz LI, Friedman PL. Supraventricular tachycardia. N Engl J Med. 1995;332:162-73.

3. Liuba I, Jönsson A, Säfström K, Walfridsson H. Gender-related differences in patients with atrioventricular nodal reentry tachycardia. Am J Cardiol. 2006;97:384-8.

4. Jackman WM, Beckman KJ, McClelland JH, Wang X, Friday KJ, Roman CA, et al. Treatment of supraventricular tachycardia due to atrioventricular nodal reentry by radiofrequency catheter ablation of slow-pathway conduction. N Engl J Med. 1992;327:313-8.

5. Ferguson JD, DiMarco JP. Contemporary management of paroxysmal supraventricular tachycardia. Circulation. 2003;107:1096-9.

6. Haissaguerre M, Gaita F, Fischer B, Commenges D, Montserrat $\mathrm{P}$, d'Ivernois $\mathrm{C}$, et al. Elimination of atrioventricular nodal reentrant tachycardia using discrete slow potentials to guide application of radiofrequency energy. Circulation. 1992;85:2162-75.

7. Uçar O, Paşaoğlu L, Ciçekçioğlu H, Vural M, Kocaoğlu I, Aydoğdu S. Persistent left superior vena cava with absent right superior vena cava: a case report and review of the literature. Cardiovasc J Afr. 2010;21:164-6.

8. Danielpour PJ, Aalberg JK, El-Ramey M, Sivina M, Wodnicki H. Persistent left superior vena cava: an incidental finding during central venous catheterization-a case report. Vasc Endovascular Surg. 2005;39:109-11.

9. Sarodia BD, Stoller JK. Persistent left superior vena cava: case report and literature review. Respir Care. 2000;45:411-6.

10. Tzeis S, Deisenhofer I, Meierhofer C, Hessling G. Two types of narrow-QRS tachycardia in a patient with coarctation of the aorta and persistent left superior vena cava. Hellenic J Cardiol. 2009;50:548-51.

11. Corîci OM, Gașpar M, Mornoș A, lancău M. Cardiac Arrhythmias in Patient with Isolated Persistent Left Superior Vena Cava. Curr Health Sci J. 2017;43:163-6. 